\title{
Les pratiques des employeurs en matière de rupture du CDI
}

Un nouveau regard sur les règles de protection de l'emploi

Employers' use of CDI terminations: a reassessment of employment protection legislation

\section{Camille Signoretto}

\section{OpenEdition}

\section{Journals}

Édition électronique

URL : http://journals.openedition.org/travailemploi/6639

DOI : 10.4000/travailemploi.6639

ISSN : 1775-416X

Éditeur

DARES - Ministère du Travail

Édition imprimée

Date de publication : 1 avril 2015

Pagination : 69-83

ISSN : 0224-4365

\section{Référence électronique}

Camille Signoretto, "Les pratiques des employeurs en matière de rupture du CDI », Travail et Emploi

[En ligne], 142 I avril-juin 2015, mis en ligne le 01 avril 2017, consulté le 22 avril 2019. URL : http:// journals.openedition.org/travailemploi/6639; DOI : 10.4000/travailemploi.6639 


\title{
Les pratiques des employeurs en matière de rupture du CDI : un nouveau regard sur les règles de protection de l'emploi ${ }^{(*)}$
}

\author{
Camille Signoretto ${ }^{(* *)}$
}

Cet article propose une lecture renouvelée des règles de protection de l'emploi à durée indéterminée en adoptant une approche microéconomique pour examiner les mouvements de main-d'auvre déclarés par les employeurs. L'objectif principal est d'enrichir la connaissance empirique sur l'usage des licenciements pour motif économique (LME) et pour motif personnel (LMP), des démissions et des ruptures conventionnelles $(R C)$. Après avoir montré les limites de la connaissance statistique actuelle, nous présentons une vision plus large de l'usage des ruptures du CDI dans les établissements d'au moins 10 salariés. Notre analyse révèle un usage très faible des licenciements économiques par les employeurs, en constante diminution depuis près de trois décennies. L'usage des LMP, lui, est plus fluctuant sur longue période, mais sa baisse depuis 2008, année d'introduction de la RC, questionne sur d'éventuelles substitutions à l'œuvre. Toutefois, ce sont plutôt des liens de complémentarités qui apparaissent entre les recours aux différents modes de rupture du CDI.

Les règles de droit du travail encadrant les ruptures des contrats à durée indéterminée (CDI) sont régulièrement décriées en France pour leur rigidité(1). L'indicateur de "protection des travailleurs permanents contre les licenciements individuels et collectifs » construit par l'OCDE place ainsi la France en $8^{\mathrm{e}}$ position des pays les plus stricts en la matière, le peloton de tête étant constitué par la Chine, l'Allemagne et la Belgique (2). Cet indicateur est utilisé pour évaluer les effets de la protection de l'emploi sur les performances du marché du travail dans une perspective macroéconomique (voir OCDE, 2004, pour une synthèse de ces effets). Pour cela, il ne s'appuie que sur les règles de droit et ne les prend en compte qu'à partir de leur seul énoncé juridique. Or, comme le soulignent Antoine LYONCAEN et Joëlle AfFichard (2008), «les règles de droit ne "font" rien si elles ne sont pas mobilisées, mises en œuvre par des acteurs» (p. 35). Suivant cette optique, nous souhaitons proposer une autre analyse de ces règles, considérant que si elles encadrent la relation d'emploi, elles ne prennent sens et effet que dans la manière dont elles sont interprétées et mobilisées par les acteurs (salariés et employeurs). L'objectif est ainsi d'approfondir la connaissance

(*) Une partie de ce travail a été soutenue par l'attribution d'une allocation postdoctorale Gestes / Région Île-de-France.

(**) Centre d'études de l'emploi; Centre d'économie de la Sorbonne (Université Paris-1 Panthéon-Sorbonne); camille. signoretto@univ-paris1.fr

(1) Le dernier débat sur une réforme des CDI et/ou des licenciements date de mars 2015.

(2) Voir les indicateurs de l'OCDE sur la protection de l'emploi : http://www.oecd.org/fr/emploi/emp/lesindicateursdelocdesurlaprotectiondelemploi.htm; consulté le 17 août 2015. empirique des différents modes de rupture du CDI (voir encadré 1) par une démarche microéconomique reposant sur une analyse des données sur les mouvements de main-d'œuvre des établissements français (EMMO-DMMO, Direction de l'animation de la recherche, des études et des statistiques [Dares], cf. infra, encadré 2) depuis la fin des années 1990, afin d'établir un panorama historique des pratiques effectives des employeurs en matière de rupture de CDI.

La littérature microéconomique portant sur les ruptures de CDI du milieu des années 1990 jusqu'au début des années 2000 a mis en avant l'augmentation des licenciements pour motif personnel et, parallèlement, la diminution des licenciements économiques. C'est sur la base de cette évolution statistique que se sont développées l'hypothèse d'un contournement des règles de droit du licenciement économique par les employeurs et, par la suite, des préconisations concernant leur assouplissement (CAHUC, KRAMARZ, 2004). Or les licenciements ne représentent qu'une part minoritaire de la rotation de la main-d'œuvre en CDI, autrement dit, des ajustements de l'emploi en CDI. En retenant une perspective temporelle plus longue, nous montrons dans cet article en quoi l'accent mis sur l'évolution opposée des deux motifs de licenciement peut donner une vision trompeuse de la protection de l'emploi à durée indéterminée. Nous y abordons plus précisément deux questions : peut-on faire apparaître des tendances structurelles dans l'évolution des ruptures de CDI, au-delà des fluctuations conjoncturelles qui les caractérisent ? En quoi la prise en compte des démissions, mais aussi des ruptures conventionnelles introduites 
récemment par le législateur, change-t-elle la représentation que donnent généralement les économistes de ces ruptures?

\section{Encadré 1 \\ Définition juridique des modes de rupture du CDI (démission, licenciements pour motif économique et pour motif personnel, rupture conventionnelle)}

Le critère permettant de distinguer la démission du licenciement repose sur la personne à l'initiative de la rupture. La démission est ainsi inscrite dans le Code du travail dans la rubrique "Rupture à l'initiative du salarié ». Le seul article (L. 1237-1) qui lui est consacré porte sur les modalités du préavis que le salarié doit respecter.

Lorsque l'initiative se situe du côté de l'employeur, pour différencier le licenciement pour motif personnel du licenciement pour motif économique, il faut déterminer qui est responsable de l'événement à l'origine du licenciement.

Si le motif du licenciement est «inhérent à la personne du salarié», alors il est dit «personnel». Le licenciement pour motif personnel (LMP) doit être «justifié par une cause réelle et sérieuse» (article L. 1232-1). Les causes fautives (faute grave ou légère) sont distinguées des causes non fautives (insuffisance professionnelle, inaptitude physique, etc.), les premières ne donnant pas les mêmes droits en termes d'indemnités de rupture (pas d'indemnité de licenciement ni d'indemnité compensatrice de préavis par exemple). Des règles de procédure, définies par le Code du travail (L. 1232-1 à L. 1232-14), encadrent le LMP.

Au contraire, si le ou les motifs de licenciement sont «non inhérents à la personne du salarié, résultant d'une suppression ou transformation d'emploi ou d'une modification, refusée par le salarié, d'un élément essentiel du contrat de travail, consécutives notamment à des difficultés économiques ou à des mutations technologiques" (L. 1233-3), il s'agit d'un licenciement pour motif économique (LME). Les procédures peuvent différer selon que le licenciement est individuel ou collectif et si l'entreprise a plus ou moins de cinquante salariés. Par exemple, le LME de dix salariés ou plus dans une même période de trente jours déclenche l'obligation de mettre en place un plan de sauvegarde de l'emploi (L. 1233-21 et suiv.).

Enfin, depuis 2008, la rupture conventionnelle $(R C)$ permet au salarié et à l'employeur «de convenir en commun des conditions de la rupture du contrat de travail qui les lie. La RC, exclusive du licenciement ou de la démission, ne peut être imposée par l'une ou l'autre des parties " (L. 123711). Elle est encadrée par une procédure formelle : possibilité d'être assisté par un tiers, délai de rétractation de quinze jours, et enfin homologation de la rupture par l'administration du Travail (unités territoriales).

Pour ce faire, nous proposons tout d'abord un état des lieux de la connaissance actuelle des modes de rupture du CDI, en en pointant les lacunes. Notamment, nous mettons en lumière la faible proportion de licenciements par rapport aux démissions dans les fins de CDI et, par conséquent, la nécessité de prendre en compte l'ensemble des modes de rupture dans une analyse de la protection de l'emploi à durée indéterminée. Sur la base de ces constats, nous réexaminons l'usage des ruptures de CDI dans les établissements d'au moins dix salariés. Nous actualisons les évolutions agrégées afin de couvrir la période plus récente (1997-2012) et de prendre en compte la rupture conventionnelle qui est apparue en 2008. Notre analyse repose ensuite sur la construction de deux indicateurs rendant compte, d'une part, du recours aux différents modes de rupture du CDI par les employeurs et, d'autre part, de l'intensité de leur usage. Ces deux indicateurs permettent de désagréger l'évolution globale en décrivant de façon plus précise les pratiques des employeurs. Pour appréhender les liens entre les différents modes de rupture et les replacer dans une optique de gestion des ressources humaines, nous proposons enfin une catégorisation des établissements selon qu'ils en font ou non un usage conjoint.

\section{Une connaissance partielle des modes de rupture du CDI et de leur évolution}

Les statistiques publiées sur les ruptures du CDI s'appuient sur deux sources de données: les mouvements de main-d'œuvre des établissements $\left(\right.$ EMMO-DMMO $\left.{ }^{(3)}\right)$ et les circonstances d'entrées des demandeurs d'emploi à l'assurance-chômage. Elles fournissent une approximation de leur nombre et des données sur la répartition des fins de CDI. Un regard est plus spécifiquement porté sur l'évolution des deux motifs de licenciement sur certaines périodes. Toutefois, comme nous le soulignons, des éléments manquent, nous semble-t-il, pour permettre une pleine compréhension des pratiques des employeurs et comparer les modes de rupture entre eux et dans le temps.

\section{Peu de licenciements parmi les fins de CDI}

Les publications trimestrielles ou annuelles de la Dares sur les mouvements de main-d'œuvre dans les établissements retracent l'évolution des modes d'entrée et de sortie des salariés à partir

(3) L'enquête trimestrielle sur les mouvements de maind'œuvre (EMMO) complète, pour les établissements de 1 à 49 salariés, les informations obtenues grâce à la déclaration mensuelle des mouvements de main-d'œuvre (DMMO) à laquelle sont assujettis tous les établissements de 50 salariés ou plus. 
de leurs taux agrégés ${ }^{(4)}$. La caractéristique principale des ruptures de CDI est leur faible intensité : en moyenne, en 2013, sur l'ensemble des mouvements de sortie tous secteurs confondus, $4,5 \%$ des salariés des établissements d'au moins un salarié ont démissionné, $1,8 \%$ a subi un licenciement «autre qu'économique» (licenciement pour motif personnel - LMP), 1,4\% a fait l'expérience d'une procédure de rupture conventionnelle (RC) et $0,6 \%$ d'un licenciement pour motif économique (LME). À l'opposé, $45 \%$ des salariés en moyenne ont connu une fin de contrat à durée déterminée (CDD) (Bourieau et al., 2014). Parmi les fins de CDI (licenciements, démissions et ruptures conventionnelles), ce sont les démissions qui constituent de loin le premier motif de rupture : elles en représentent $54 \%$ en 2013. Viennent ensuite les LMP (22\% des fins de $\mathrm{CDI})$, les ruptures conventionnelles (17\%) et enfin les LME (7\%). Ces taux diffèrent selon le secteur et la taille des établissements. Les démissions sont ainsi plus fréquentes dans le secteur tertiaire que dans l'industrie, alors que les LMP et les RC sont plus courants dans la construction et le tertiaire, les LME l'étant enfin dans l'industrie. Les taux agrégés pour les différentes ruptures diminuent globalement avec la taille d'effectifs des établissements, sauf pour le LMP dont le taux est le plus faible dans les établissements de 1 à 9 salariés en 2013 (BouriEAU et al., 2014).

Ces publications de la Dares donnent une vision d'ensemble des ruptures, mais ne fournissent pas d'informations sur leur nombre. Pour cela, il faut se référer à une autre source statistique : les circonstances d'entrée des demandeurs d'emploi lors de leur inscription à l'assurance-chômage. En 2013, on dénombre ainsi 503600 inscriptions après un LMP (catégorie «autres licenciements»), 262550 après une RC, 154900 après un LME, et 168500 après une démission (5). Cependant, ces chiffres ne donnent que des ordres de grandeur approximatifs des modes de rupture, en raison même du champ de cette source statistique. En effet, tous les individus ne s'inscrivent pas à l'assurance-chômage après une rupture de leur contrat de travail( ${ }^{(6)}$. Les démissions sont donc largement sous-estimées; les autres modes de rupture, licenciements et ruptures

(4) Par exemple, le taux agrégé de démissions rapporte le nombre total de démissions observées dans tous les établissements à la somme des effectifs salariés moyens de tous les établissements

(5) Voir le fichier de données des «séries mensuelles nationales sur les demandeurs d'emploi inscrits et les offres collectées par Pôle emploi» disponible sur le site internet de la Dares à l'adresse suivante : http://travail-emploi. gouv.fr/etudes-recherches-statistiques-de,76/statistiques,78/ chomage,79/les-demandeurs-d-emploi-inscrits-a,264.html ; consulté le 17 août 2015.

(6) Certains peuvent en effet avoir retrouvé un emploi immédiatement après leur départ, tandis que d'autres ne peuvent pas bénéficier des allocations chômage (salariés démissionnaires ou jeunes n'ayant pas ouvert leurs droits). conventionnelles, l'étant également mais dans une moindre mesure.

Quoi qu'il en soit, ces informations statistiques indiquent que la rotation de la main-d'œuvre en CDI consiste majoritairement en des départs volontaires des salariés (démissions), alors que les licenciements (surtout ceux pour motif économique) occupent une place moindre.

\section{Une évolution opposée entre LMP et LME qui apparaît... sur la seule période 1997-2003}

Alors que la part des licenciements au sein des fins de CDI est somme toute assez faible, c'est pourtant sur leur évolution que s'est focalisée l'attention des analyses statistiques. Au cours des années 2000, deux études portant sur les mouvements de maind'œuvre des établissements d'au moins cinquante salariés mettent en évidence une évolution différente des deux motifs de licenciement : la première pointe la baisse du licenciement économique entre 1983 et 2000, même si l'évolution est marquée par des fluctuations conjoncturelles (TomAsinI, LE Roux, 2002), alors que la seconde montre une augmentation des LMP de plus de $70 \%$ et une diminution des LME d'environ $60 \%$ entre 1988 et 2003 (7) (Lagarenne, Le Roux, 2006). Plus précisément, la hausse du LMP n'apparaît continue que depuis 1997 puisqu'à cette date, le niveau des LMP est en réalité le même qu'en 1988, alors que le LME ne retrouve jamais son niveau initial sur la période 1988-2003. Cette évolution opposée des deux motifs de licenciement est également mise en avant dans d'autres études s'appuyant sur les motifs d'entrée à l'assurance-chômage (CAhuc, Kramaz, 2004; Serverin et al., 2008). C'est plus précisément à partir du milieu des années 1990 (autour de 1995-1997) que les deux courbes correspondant aux deux motifs de licenciement se croisent : les LMP augmentent alors que les LME diminuent, et ce jusqu'en 2003-2005. Cette observation s'est imposée comme le principal fait stylisé de la littérature économique et gestionnaire sur les licenciements. Elle a donné lieu à plusieurs explications.

Une première hypothèse explique la substitution du LME par le LMP par une logique de contournement du droit par les employeurs. Elle s'appuie théoriquement, même si le plus souvent implicitement, sur les modèles d'économie du droit qui mettent en évidence un usage stratégique des

(7) Notons que sur une période antérieure, c'est une évolution contraire qui était observée à partir des motifs d'entrée à l'assurance-chômage : entre 1976 et 1980, une baisse des LMP de presque $20 \%$ s'oppose alors à une hausse des LME de $40 \%$ (Colin et Welcomme, 1981). 
règles juridiques encadrant les licenciements ${ }^{(8)}$. Les employeurs substitueraient des licenciements pour motif personnel à des licenciements pour motif économique parce qu'ils considèrent les procédures régissant le LME plus contraignantes, plus complexes (règles supplémentaires pour les licenciements économiques collectifs) et plus incertaines que celles encadrant le LMP (Lagarenne, Le Roux, 2006; Cahuc, Kramarz, 2004; Pignoni, Zouary, 2003; Palpacuer et al., 2005). Une seconde hypothèse met en avant les effets des nouvelles pratiques de gestion des ressources humaines (GRH) dans les entreprises. Elle explique la montée du LMP par la diffusion de pratiques de gestion plus individualisées et liées aux formes de management par objectifs reposant, notamment, sur des entretiens d'appréciation ou d'évaluation (PIGNONI, ZoUARY, 2003; Palpacuer et al., 2005). De ces deux explications possibles, c'est surtout la première qui s'est imposée dans la littérature et le débat public, alors même que les éléments empiriques ne permettent pas de trancher véritablement. Sur données quantitatives, Évelyne SERVERIN et ses coauteurs (2008) concluent plutôt à un rejet de cette hypothèse car les comportements d'embauche assez dynamiques observés chez des employeurs ayant procédé à un LMP ne permettent pas d'étayer un usage de ce type de licenciement en lieu et place d'un licenciement pour motif économique ${ }^{(9)}$. En revanche, sur données qualitatives et à partir d'un échantillon non représentatif, Florence PALPACUER et ses coauteurs (2005) identifient auprès de certains employeurs les arguments sous-tendant l'hypothèse de substitution: procédure du LMP moins contraignante ${ }^{(10)}$ et durcissement jurisprudentiel sur le motif économique.

Ainsi, ces analyses pointent une évolution opposée des deux motifs de licenciement, qui ne s'observe pourtant que sur une période de sept à huit ans, puisque le LMP n'augmente de manière continue que depuis 1997 et commence à stagner dès 2003-2004, alors que le LME, lui, semble diminuer depuis le début des années 1980. Cette évolution n'est donc peut-être qu'un phénomène ponctuel qui ne représente qu'une partie des changements

(8) «Les entreprises et les salariés vont probablement utiliser de manière stratégique la législation sur les licenciements, créant un modèle caractéristique de la distribution des licenciements par types juridiques. Ces modèles vont sûrement différer entre les pays, en raison des différentes mesures incitatives prévues dans les cadres juridiques nationaux sur l'utilisation des licenciements individuel et collectif, ou même sur l'utilisation des différents motifs de licenciement individuel (par exemple pour motif personnel ou pour motif économique).» (GARCíAMartínez, Malo [2007], p. 152, traduction de l'auteure.)

(9) Dans le cas d'un motif économique, le poste laissé vacant ne devrait pas en effet être pourvu puisque l'emploi était supprimé.

(10) Sont notamment invoquées, dans le cas du LME, l'obligation d'information des institutions représentatives du personnel et des pouvoirs publics, l'obligation de reclassement, l'interdiction d'une nouvelle embauche sur le même poste pendant six mois. à l'œuvre dans les pratiques des employeurs en matière de rupture du CDI depuis trente ans.

\section{Les limites des sources statistiques}

Pour bien comprendre et interpréter les constats statistiques mis en avant par les deux sources de données mobilisées sur les ruptures du CDI, il est nécessaire de revenir brièvement sur la manière dont les catégories statistiques sont construites. Historiquement, le dénombrement des modes de rupture n'a jamais existé de manière exhaustive, sauf pour les ruptures qui, légalement, doivent faire l'objet d'une procédure d'information ou de demande d'autorisation auprès de l'administration du Travail (les LME entre 1975 et 1986; les RC, les plans de sauvegarde de l'emploi et les licenciements des salariés «protégés ${ }^{(11)}$ 》 aujourd'hui). Outre la question de l'exhaustivité, l'appareil statistique est aujourd'hui confronté à un problème d'identification des licenciements économiques. En effet, durant les trois dernières décennies, les ruptures ayant des raisons économiques se sont progressivement diversifiées, s'éloignant ainsi de leur seule qualification en licenciement pour motif économique : elles peuvent désormais prendre la forme de départs volontaires avec résiliation du contrat à l'amiable pour motif économique, de ruptures d'un commun accord après l'entrée dans un dispositif public d'accompagnement de restructuration ${ }^{(12)}$ ou encore de ruptures conventionnelles dont la cause économique est aujourd'hui admissible(13) (SignORETTO, 2015). Or, on ne sait pas bien où ces modes de rupture sont classés statistiquement, que ce soit dans les motifs d'entrée à Pôle emploi ou lors des déclarations administratives des employeurs dans les EMMODMMO. Dans le premier cas par exemple, un salarié bénéficiaire d'un contrat de sécurisation professionnelle se voit inscrit en catégorie $\mathrm{D}$, «stagiaire de la formation professionnelle», à Pôle emploi. Comme ce sont sur les seules catégories $\mathrm{A}, \mathrm{B}$ et $\mathrm{C}$ que portent les statistiques des motifs d'entrée, cela tend à sousestimer le dénombrement des LME à partir de cette source statistique ${ }^{(14)}$, tout particulièrement depuis les années 2000 (SignoretTo, 2013a).

(11) Les représentants du personnel ou les médecins du travail par exemple.

(12) Créés par la loi ou sous l'impulsion des pouvoirs publics dès le milieu des années 1980, les dispositifs publics d'accompagnement des restructurations sont proposés par les entreprises de manière volontaire ou obligatoire lorsqu'elles envisagent de procéder à des licenciements pour motif économique, ou qu'elles anticipent des changements de la structure de leurs emplois. Il s'agit principalement aujourd'hui du contrat de sécurisation professionnelle, créé en 2011 à la suite de la fusion entre la convention de reclassement personnalisé et le contrat de transition professionnelle. Voir SignoretTo (2013a) pour une présentation détaillée de ces dispositifs.

(13) Voir l'arrêt de la Cour de cassation du 9 mars 2011.

(14) Toutefois, à l'issue du dispositif (douze mois pour le contrat de sécurisation professionnelle) et s'il n'a pas retrouvé d'emploi, le bénéficiaire d'un tel contrat peut basculer en catégories A, B ou C, sous le motif d'entrée «autres cas». 
Enfin, en plus des difficultés de dénombrement et d'identification des modes de rupture, d'autres questions empiriques restent en suspens : combien d'établissements recourent chaque année aux différents modes de rupture du CDI ? Quelle est l'évolution de ces ruptures depuis le milieu des années 2000 et, notamment, celle des licenciements par rapport aux démissions et aux RC ? Comment $\mathrm{y}$ distinguer des tendances longues, au-delà des fluctuations conjoncturelles ? À partir des EMMODMMO (voir encadré 2), qui apparaissent comme la source statistique la plus complète et la moins dépendante de changements de règles administratives, nous proposons dans ce qui suit d'apporter des éléments de réponse à ces questions.

\section{Encadré 2 \\ L'utilisation des EMMO-DMMO}

Nous utilisons les données sur les mouvements de main-d'œuvre, les EMMO et DMMO de la Dares, de deux manières : d'une part, à partir des taux agrégés diffusés sur le site de la Dares ${ }^{(1)}$ sur la période 1997-2012 et, d'autre part, à partir des fichiers bruts obtenus auprès de la Dares sur la période 1997-2010. Ces fichiers nous ont permis de construire une base de données qui couvre les mêmes champs sectoriels que ceux des études de la Dares (industrie, construction, tertiaire marchand et non marchand, hors secteur agricole) et porte sur l'ensemble des établissements d'au moins dix salariés (échantillon exhaustif pour les DMMO, et représentatif pour les EMMO en utilisant les pondérations) pour la période 1997-2010, date jusqu'à laquelle nous avons pu accéder aux données.

(1) Consultables à l'adresse suivante : http://travail-emploi. gouv.fr/etudes-recherches-statistiques-de,76/statistiques,78/ emploi,82/les-mouvements-de-main-d-oeuvre,272/ les-donnees-sur-les-mouvements-de,2268/les-donnees-surles-mouvements-de,2633.html; consultée le 17 août 2015.

\section{Deux indicateurs pour préciser l'usage des modes de rupture du CDI au-delà de l'intensité agrégée}

Les statistiques présentées jusqu'à maintenant nous ont donné des informations générales sur les modes de rupture, mais n'ont pas décrit les pratiques concrètes des employeurs et l'usage (15) qu'ils font de ces modes de rupture. Pour caractériser cet usage des ruptures de CDI dans les établissements au-delà de

(15) Nous utilisons le terme d'«usage» principalement pour les licenciements puisque ce sont ces ruptures qui relèvent d'une volonté des établissements. Pour les démissions, nous parlerons plutôt de «présence» dans les établissements. l'intensité évaluée au niveau agrégé, nous proposons deux indicateurs : l'un mesurant le recours à chaque mode de rupture dans les établissements; l'autre indiquant l'intensité mesurée, au niveau individuel, de l'usage de chaque mode de rupture pour les seuls établissements y recourant (voir encadré 3).

\section{Encadré 3}

\section{Indicateurs de recours et d'intensité des modes de rupture du CDI}

Le premier indicateur rend compte du recours à chaque mode de rupture par les employeurs et ce, pour une année donnée. II repose sur la part des établissements qui ont utilisé au moins une fois au cours d'une année l'un des modes de rupture. II permet d'analyser des phénomènes plus structurels, tels que des processus de diffusion (dans le sens de généralisation) ou, au contraire, d'abandon de l'usage d'un mode de rupture.

Le deuxième indicateur décrit l'intensité avec laquelle les établissements recourent à chaque mode de rupture du CDI. Nous calculons des taux individuels rapportant le nombre d'occurrences d'un mode de rupture donné à l'effectif salarié moyen de l'établissement, pour les seuls établissements y recourant, puis nous mesurons la moyenne de ces taux individuels. II se distingue des taux agrégés établis par la Dares dans la mesure où il donne un poids identique à chaque établissement quelle que soit sa taille (ce sont les grands établissements qui ont des taux plus faibles, ce qui tend à réduire le taux agrégé par rapport à la moyenne des taux individuels) et qu'il ne porte que sur les établissements recourant effectivement au mode de rupture considéré. Cet indicateur d'intensité permet de mesurer l'ampleur de l'usage des différents modes de rupture et d'observer ainsi les possibles fluctuations conjoncturelles.

\section{Un très faible recours aux licenciements économiques mais une intensité plus forte par rapport aux LMP et aux RC}

À notre connaissance, seules deux études portant sur les années 1990 contiennent des informations relatives au nombre d'établissements qui recourent aux licenciements. En 1992, le recours au licenciement économique concerne ainsi $31 \%$ des établissements d'au moins cinquante salariés (LE Minez, Maurin, 1994), alors qu'en 2000 la part est de $12,5 \%$ pour les établissements d'au moins dix salariés du secteur privé industriel et commercial ${ }^{(16)}$ (Tomasini, Le Roux, 2002). Comme le montre le graphique 1, cette part est de nouveau divisée par deux, pour atteindre $6 \%$ des établissements d'au moins dix salariés en 2010. La diminution du recours au LME a donc été très forte en une

(16) Cette part varie entre 15 et $20 \%$ pour les établissements d'au moins cinquante salariés. 
vingtaine d'années, de telle sorte qu'aujourd'hui ce mode de rupture n'est utilisé que par un nombre très restreint d'établissements. Quant aux autres modes de rupture du CDI, les établissements sont plus nombreux à y recourir : $33 \%$ pour le LMP et $22 \%$ pour la RC. En outre, $61 \%$ d'entre eux connaissent des démissions en 2010.

\section{Graphique 1 : Recours et intensité des ruptures du CDI en 2010}

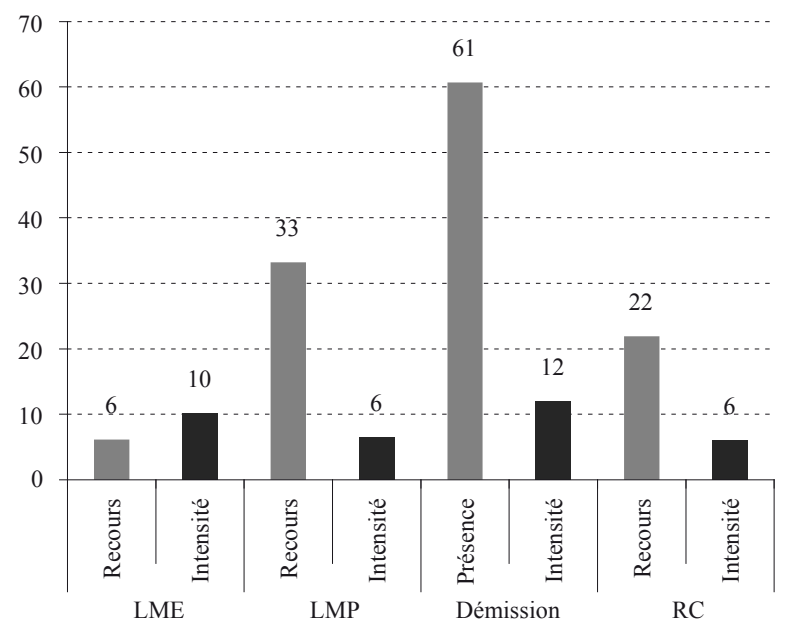

Lecture : En 2010, 6\% des établissements d'au moins dix salariés ont eu recours au licenciement pour motif économique. Ces mêmes établissements ont licencié en moyenne $10 \%$ de leurs effectifs salariés pour ce motif.

Champ : Établissements de dix salariés ou plus du secteur concurrentiel (hors agricole) de la France métropolitaine.

Source : Calculs de l'auteure à partir des EMMO-DMMO, Dares, données pondérées.

Concernant l'intensité, le graphique 1 montre que les $6 \%$ des établissements utilisateurs du LME en 2010 ont licencié en moyenne $10 \%$ de leurs effectifs salariés pour ce motif, ce qui est bien plus élevé que le taux agrégé produit par la Dares $(0,6 \%$ en 2010). Ce taux de LME apparaît également supérieur à l'intensité moyenne individuelle des LMP et des RC pour les établissements utilisateurs de ces ruptures (6\%). Le LME s'inscrit en effet plus souvent dans le cadre de vastes plans de réductions d'effectifs; la différence d'intensité avec les autres modes de rupture reste toutefois assez faible. Enfin, c'est pour les établissements qui connaissent des démissions que l'intensité d'usage est la plus forte (12\%).

Ces résultats statistiques confirment la place des démissions dans les ruptures du CDI, tant par la quantité d'établissements touchés que par leur intensité au sein de ces établissements. Au contraire, les licenciements pour motif économique, encadrés, rappelons-le, par des règles juridiques critiquées pour leur complexité et leur rigidité, sont caractérisés par une très faible présence dans les établissements.

\section{Des spécificités par secteur et taille d'effectifs différentes selon l'indicateur d'usage mobilisé}

Comme nous l'avons observé pour les statistiques agrégées des différents modes de rupture du CDI, il existe de fortes spécificités selon les secteurs d'activité et les tailles d'effectifs des établissements. L'analyse désagrégée permet de mettre en lumière de nouvelles particularités selon l'indicateur de recours ou d'intensité.

On retrouve ainsi en 2010 un recours plus important du LME dans le secteur industriel, mais son intensité semble aussi forte dans la construction que

Graphique 2 : Recours et intensité des ruptures du CDI par secteur d'activité en 2010
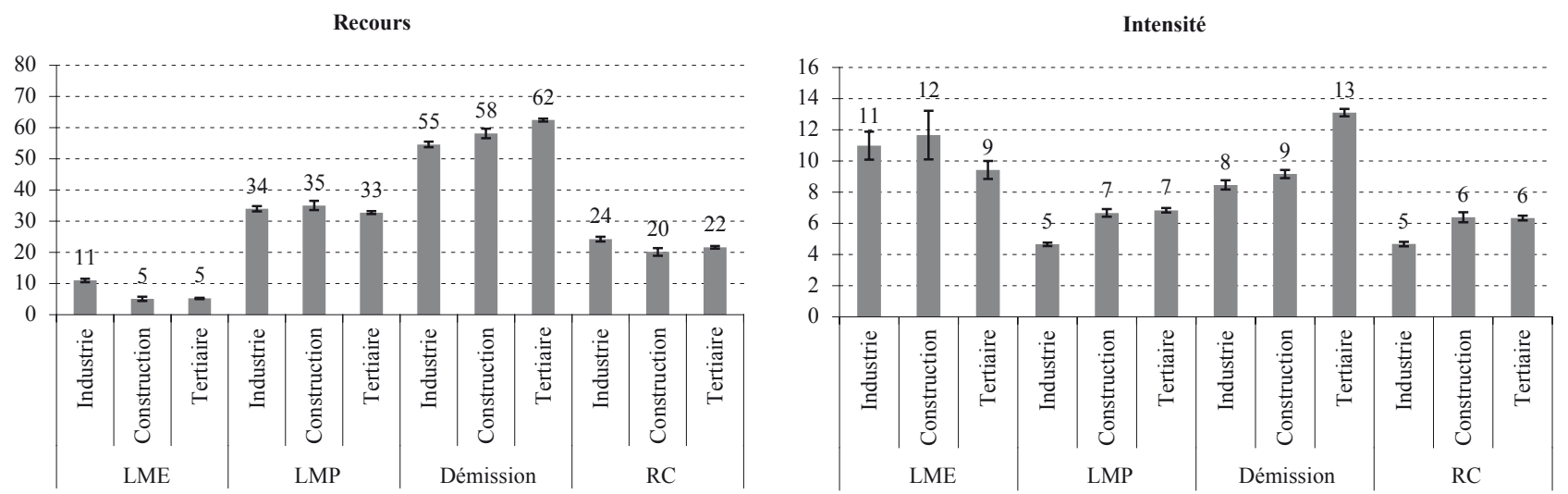

Note : Les intervalles de confiance à $95 \%$ des moyennes sont indiqués sur chaque colonne.

Lecture : $34 \%$ des établissements du secteur industriel ont recouru au licenciement pour motif personnel en 2010. Ces mêmes établissements ont licencié en moyenne $5 \%$ de leurs effectifs salariés pour ce motif.

Champ : Établissements de dix salariés ou plus du secteur concurrentiel (hors agricole) de la France métropolitaine.

Source : Calculs de l'auteure à partir des EMMO-DMMO, Dares, données pondérées. 
Graphique 3 : Recours et intensité des ruptures du CDI par taille d'effectifs en 2010
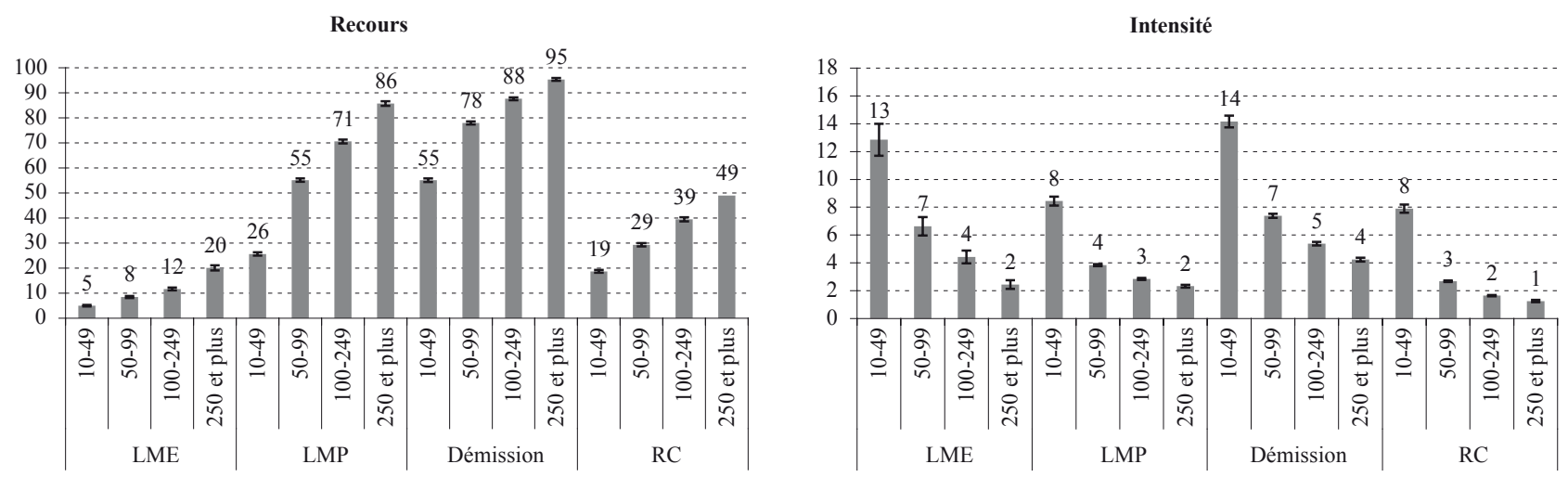

Note : Les intervalles de confiance à $95 \%$ des moyennes sont indiqués sur chaque colonne.

Lecture : $29 \%$ des établissements de cinquante à quatre-vingt-dix-neuf salariés ont conclu des ruptures conventionnelles en 2010. Ces mêmes établissements se sont séparés en moyenne de $3 \%$ de leurs effectifs par le biais de ce mode de rupture.

Champ : Établissements de dix salariés ou plus du secteur concurrentiel (hors agricole) de la France métropolitaine.

Source : Calculs de l'auteure à partir des EMMO-DMMO, Dares, données pondérées.

dans l'industrie ${ }^{(17)}$ (voir graphique 2). Le constat inverse est observé pour les LMP : une intensité plus importante dans les secteurs de la construction et du tertiaire, confirmant les études précédentes, mais un recours équivalent dans l'industrie et dans la construction. Concernant les démissions, c'est bien dans le tertiaire que leur présence et leur intensité sont les plus importantes. Enfin, l'intensité de la $\mathrm{RC}$ est la plus soutenue dans la construction et le tertiaire mais, là encore, son recours est légèrement plus élevé dans l'industrie. L'analyse sectorielle est intéressante à plusieurs égards : il existe finalement peu d'écarts dans l'intensité et le recours aux modes de rupture entre les secteurs, excepté pour les démissions; et on observe des usages différents des ruptures selon l'intensité et le recours entre les secteurs (par exemple entre RC et LMP dans l'industrie).

Si l'on s'intéresse à présent à la taille des établissements (voir graphique 3), on vérifie que l'intensité de l'usage des ruptures diminue à mesure que la taille des établissements s'accroît. En revanche, plus un établissement est grand, plus il est en situation de mettre fin à au moins un CDI (taux de recours élevé). Par exemple, une grande majorité des établissements de 250 salariés et plus connaissent des démissions et recourent aux LMP (respectivement $95 \%$ et $86 \%$ ), alors qu'ils sont un sur deux à avoir conclu au moins une fois une rupture conventionnelle en 2010. Pour ce qui est de l'intensité des ruptures, l'effet taille est particulièrement marqué pour les LME et les démissions, alors qu'il l'est moins pour les LMP et les RC. Quant au recours, l'effet taille est important pour les LMP, mais assez

(17) Pour estimer la précision des deux indicateurs, nous avons calculé les intervalles de confiance des moyennes (indiqués dans les tableaux et/ou graphiques lorsque cela était pertinent). Dans le cas où les intervalles de confiance ne se chevauchent pas, cela permet de donner une indication sur la significativité de la différence de moyennes entre deux groupes d'établissements ou entre deux années. faible pour les LME. Le profil d'usage des modes de rupture selon la taille est finalement proche entre les LMP et les RC concernant l'intensité à laquelle les établissements y recourent. À noter également que la part des établissements concluant une rupture conventionnelle est relativement élevée dès les établissements de dix à quarante-neuf salariés, de manière comparable au profil d'usage des LMP chez ces petits établissements.

\section{Quel(s) changement(s) de l'évolution des ruptures du CDI depuis le milieu des années 2000 ?}

Dans l'objectif de questionner le caractère structurel, c'est-à-dire sur longue période, de l'opposition entre l'usage des deux motifs de licenciement, nous présentons dans un premier temps l'évolution agrégée des modes de rupture entre 1997 et 2012. Il s'agit ensuite de décomposer ces évolutions pour comprendre si les tendances observées de manière agrégée sont dues à une évolution de l'intensité avec laquelle les établissements recourent au mode de rupture (des mêmes établissements les utilisent plus ou moins intensément), ou plutôt à une évolution de la diffusion de l'usage de ces ruptures dans les établissements (plus ou moins d'établissements $\mathrm{y}$ ont recours).

\section{Une baisse structurelle du LME et une baisse du LMP depuis 2008 concomitante de la progression rapide de la RC}

Repartant de l'année 1997 à partir de laquelle les courbes des deux motifs de licenciement se croisent, il s'agit de proposer une vision de plus long terme incluant la période récente et la rupture conventionnelle créée en 2008. L'évolution des ruptures de CDI est donc prolongée jusqu'en 2012, à partir des taux agrégés de rupture produits par la Dares pour les 
établissements d'au moins dix salariés ${ }^{(18)}$. Le choix a été fait de comparer cette évolution en prenant 2009 comme année de référence, d'une part, parce que c'est la première année d'utilisation complète de la RC et, d'autre part, parce qu'elle correspond à l'année de pic de la dernière crise économique. Que nous apprend alors le graphique 4 par rapport aux évolutions connues jusqu'ici?

\section{La baisse structurelle du LME et le caractère conjoncturel des démissions}

Tout d'abord, il ressort de nouveau une forte baisse du licenciement économique sur l'ensemble de la période (- 70\% entre 1997 et 2012), même si un pic est observé pour 2009, dont le niveau reste toutefois en dessous de celui de l'année 1997. Si l'on reprend la tendance observée depuis le début des années 1980, cela signifie que les LME sont de moins en moins nombreux depuis près de trente ans, même s'ils restent sensibles aux fluctuations conjoncturelles (pics en 2001-2003 et 2008-2009). Sur une longue période, le caractère structurel de la baisse du LME apparaît donc de manière indiscutable et son évolution est par là déconnectée de celle du LMP, ce qui remet en cause une lecture de l'évolution des deux motifs de licenciement uniquement en termes de substitution de l'un par l'autre. Cette baisse peut s'expliquer par les changements de pratiques des entreprises en matière de suppressions d'emplois, qui peuvent de plus en plus prendre une diversité de formes juridiques : fins de CDD ou de missions d'intérim, départs en retraite, mobilités internes au sein d'une entreprise ou d'un groupe, résiliations à l'amiable, etc. (IRES, 2009; BEAUJoLINBellet, Schmidt, 2012; Signoretto, 2015). Et elle peut renvoyer aux difficultés d'identification statistique que ces pratiques engendrent.

Par ailleurs, c'est avec les démissions qu'il y a une opposition des évolutions observées dans la mesure où LME et démission sont les deux modes de rupture les plus sensibles à la conjoncture économique (ARNold, 2009). Par exemple, dans les phases de croissance (1997-2001, 2010-2011), les démissions augmentent tandis que les LME diminuent, alors que durant les phases de ralentissement conjoncturel ou de crise (2001-2003, 2008-2009), c'est le contraire qui est constaté. Damien SAuze (2003) et Florent NoËL (1995) avaient déjà fait apparaître à un niveau agrégé des effets de compensation entre les licenciements économiques et les

(18) Ces taux ont été récupérés sur le site internet de la Dares pour la période 1999-2012, et sur un document d'études de la Dares (LuTinier, 2007) pour les années 1997 et 1998. Le champ des établissements est donc plus large que celui utilisé par Christine Lagarenne et Marine Le Roux (2006), qui ne portait que sur les établissements d'au moins 50 salariés. Toutefois, les tendances restent similaires si l'on ne prend en compte que les grands établissements. démissions dans un contexte de fort ralentissement de l'activité économique.

Graphique 4 : Évolution des modes de rupture du CDI entre 1997 et 2012 (base 100 : 2009)

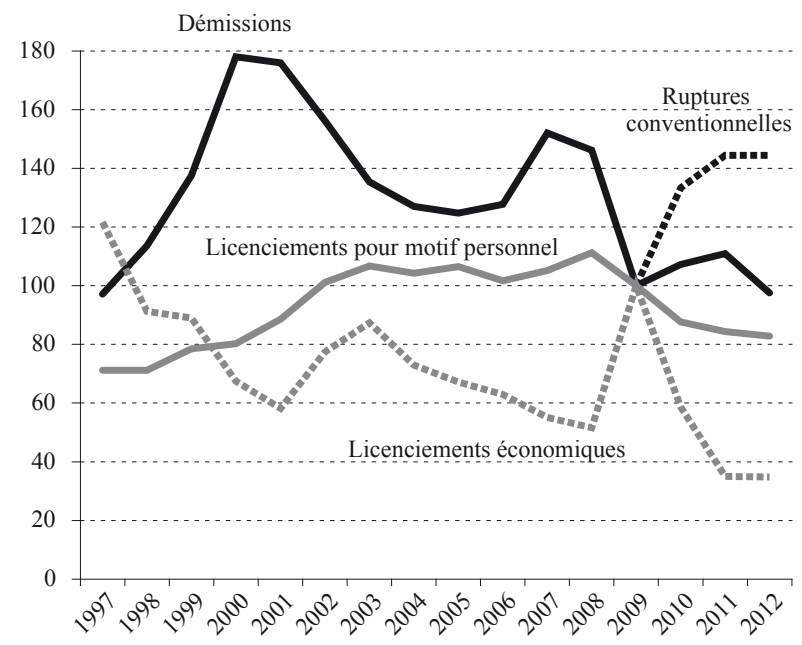

Lecture : Entre 2009 (base 100) et 2012, les licenciements pour motif économique ont diminué d'environ $65 \%$.

Champ : Établissements d'au moins dix salariés du secteur concurrentiel (hors agricole) de la France métropolitaine. Rupture conventionnelle : établissements d'au moins un salarié

Source : Calculs de l'auteure à partir des taux de sorties des EMMODMMO, Dares.

\section{Les trois "phases" du LMP et la progression rapide de la $R C$}

L'usage des LMP progresse de 1997 à 2003 (+50\%), confirmant les observations de LAGARENNE et LE Roux sur les grands établissements. Toutefois, après cette date, le LMP fluctue très peu, de sorte qu'en 2009 il retrouve son niveau de 2002, et commence à diminuer dès 2008 (de près de $26 \%$ entre 2008 et 2012). L'évolution du LMP semble donc paradoxalement plus fluctuante que celle du LME sur les trente dernières années - légère baisse à la fin des années 1970, forte augmentation à la fin des années 1990 et au début des années 2000, stagnation ensuite et baisse continue depuis 2008 -, même s'il semble avoir légèrement progressé globalement (+16\% entre 1997 et 2012).

Mais surtout, la baisse observée depuis 2008 coïncide avec l'entrée en vigueur de la rupture conventionnelle. Or le graphique 4 montre une progression très forte de ce mode de rupture entre 2009 et 2012 (près de 45\%). L'hypothèse de la substitution du LMP par la RC apparaît donc comme centrale pour expliquer la baisse du LMP. Comme la RC est un mode de rupture plus attrayant pour les employeurs (absence de motif à énoncer, absence de préavis, faible risque de contestation judiciaire) et les salariés (droit aux allocations chômage), on peut en effet s'attendre à ce qu'elle se substitue à l'ensemble des modes de rupture du CDI déjà existants. L'évolution statistique des LMP et des RC entre 2009 et 2012 peut s'interpréter en ce sens, ainsi que celle des LME et des RC. 


\section{Graphique 5 : Évolution du recours et de l'intensité des modes de rupture du CDI}
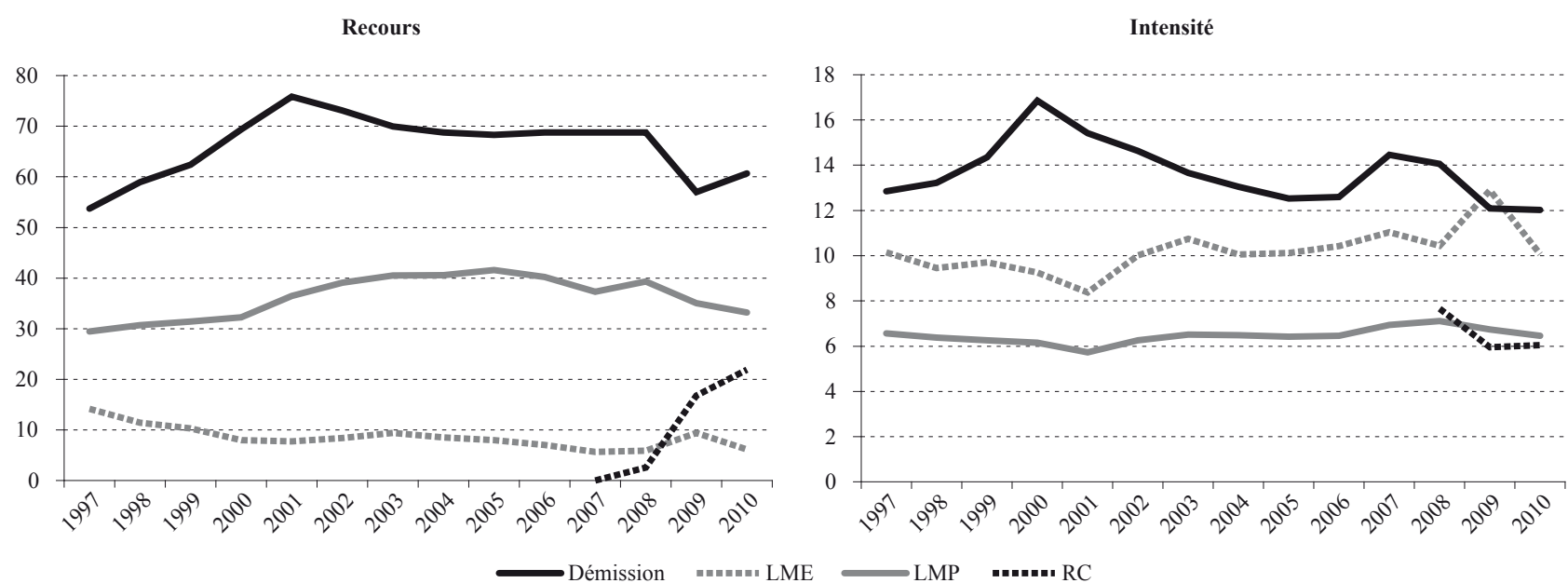

Lecture : En 2010, plus de 20\% des établissements d'au moins dix salariés ont eu recours à au moins une rupture conventionnelle, à un taux moyen de $6 \%$.

Champ : Établissements de dix salariés ou plus du secteur concurrentiel (hors agricole) de la France métropolitaine.

Source : Calculs de l'auteure à partir des EMMO-DMMO, Dares, données pondérées.

Toutefois, il reste difficile de tirer des conclusions de ces simples évolutions statistiques, tant il y a d'éléments à prendre en considération, en premier lieu la conjoncture économique particulière de la période. Seule une analyse économétrique aboutie pourrait permettre de contrôler ces éléments. À partir d'un échantillon d'entreprises obtenu par l'appariement de deux bases de données (les EMMO-DMMO et les enquêtes annuelles d'entreprises - EAE-Fare ${ }^{(19)}$ - rassemblant des variables économiques et comptables), une autre étude (Signoretto, 2013b) révèle l'absence de comportement de substitution entre les LMP et les RC entre 2008 et 2009, ainsi qu'entre les démissions et les RC. C'est plutôt un usage des RC pour un motif économique qui semble ressortir de la période, suggérant des possibles substitutions du LME par la $\mathrm{RC}$ sur cette période.

Au-delà de ces éléments empiriques, la question de la substitution entre la RC et les autres modes de rupture du CDI est plus complexe que celle apparue entre les deux motifs de licenciement au début des années 2000. En effet, la RC étant une rupture d'un commun accord, le comportement de l'employeur sera dépendant de celui du salarié (et vice versa). Même si l'employeur veut substituer une RC à un licenciement, l'accord du salarié lui est nécessaire. De plus, si substitution il y a, elle est tout à fait légale dans la mesure où la $\mathrm{RC}$ devient une alternative de rupture pour les employeurs et les salariés, et que de nombreuses situations de recours ont été reconnues possibles par la jurisprudence (cause économique, litige préalable entre l'employeur et le salarié, etc.).

(19) Fare : Fichier approché des résultats Esane (Élaboration des statistiques annuelles d'entreprise).

\section{Des changements dans le recours aux modes de rupture par les employeurs plutôt que dans leur intensité}

Sur la période 1997-2010 (voir encadré 2), c'est d'abord l'évolution du recours aux modes de rupture du CDI qui explique celle constatée au niveau agrégé, l'intensité étant plutôt stable, ou en légère baisse (voir graphique 5). Le licenciement économique est par exemple nettement moins utilisé dans les établissements entre 1997 et 2010 (- 56\%), mais pour ceux qui continuent à y recourir, ils le font en moyenne avec une même intensité. Ce constat statistique permet ainsi d'étayer l'argument d'un abandon progressif du licenciement économique comme forme juridique de rupture retenue par les employeurs. Au contraire, les LMP se sont légèrement diffusés dans les établissements $(+13 \%)$, mais là encore, l'intensité à laquelle les établissements ont licencié pour ce motif reste stable sur la période. Seules les démissions ont des évolutions différentes selon l'indicateur : davantage d'établissements connaissent des démissions $(+13 \%)$, mais de façon moins intensive (- 6\%). Enfin, confirmant l'évolution agrégée, l'usage des RC progresse rapidement (recours comme intensité).

Ces graphiques font également apparaître une évolution de l'usage des LMP relativement indépendante des autres modes de rupture. Entre 1997 et 2003, le recours au LMP augmente en effet de manière continue $(+37 \%)$, avec une accélération à partir de l'année 2000. Après 2003, il fluctue et, surtout, baisse à deux reprises: entre 2005 et $2007(-10 \%)$ et entre 2008 et $2010(-16 \%)$. Si la baisse constatée à partir de 2008 peut être légitimement mise en parallèle avec la progression de la RC comme nous l'avons fait précédemment, les pratiques des employeurs en matière de recours au LMP ont en réalité vraisemblablement commencé 
à se modifier avant l'introduction de cette dernière. Ce phénomène n'était pas visible dans l'évolution agrégée (voir graphique 1), parce que cette baisse du recours au LMP entre 2005 et 2007 s'oppose à une hausse de son intensité sur ces mêmes années. Toutefois, il reste difficile à ce stade et sans un travail qualitatif plus poussé sur les pratiques de gestion des ressources humaines des employeurs, de comprendre cet usage particulier des LMP dès $2005^{(20)}$

\section{Des effets de structure sectorielle?}

Alors que les pratiques de gestion de la maind'œuvre diffèrent entre les secteurs d'activité (voir supra) et que la répartition sectorielle des établissements s'est largement modifiée ces dernières décennies (diminution du poids de l'industrie, développement du tertiaire), une analyse désagrégée de l'usage des ruptures paraît ici nécessaire pour saisir si l'évolution de l'intensité et du recours aux modes de rupture n'est pas expliquée par d'éventuel(s) changement(s) de structure des établissements par secteur d'activité(21).

$\mathrm{Au}$ début de notre période d'étude, le secteur industriel est caractérisé par un recours au licenciement économique plus développé, alors que le secteur tertiaire se distingue par des taux de démission et de LMP plus élevés (voir tableau en annexe). La diminution du poids du secteur industriel pourrait donc avoir entraîné avec elle la baisse de l'usage du licenciement économique. De même, il est possible que le développement du secteur tertiaire ait «tiré vers le haut» le recours aux LMP et aux démissions. Mais, en réalité, on observe peu de différence entre l'évolution générale et l'évolution sans changement de structure par secteur d'activité (voir tableau en annexe). Plus précisément, le changement de structure a légèrement accéléré la diminution du recours aux LME (- $56 \%$ vs - 53\%), alors qu'il a freiné la baisse de l'intensité des LMP et des démissions (stable $v s-4 \%$ pour le LMP; - 6\% vs - 10\% pour les démissions). Ainsi, ce n'est pas la contraction du secteur industriel qui explique le recul de l'usage du LME dans les établissements, mais bien

(20) Pour tenter néanmoins de comprendre le ralentissement du recours au LMP dès 2003, l'hypothèse d'un changement de pratique de GRH avancée pour expliquer la hausse du LMP à la fin des années 1990 peut être de nouveau mobilisée ici. Le recours à des LMP pour insuffisance de résultat ou non-atteinte des objectifs serait désormais moindre, du fait d'une évolution de la jurisprudence au début des années 2000. Celle-ci semble en effet exiger une justification plus rigoureuse de la cause d'insuffisance de résultats ainsi qu'en témoignent plusieurs arrêts de la Cour de cassation (voir notamment les arrêts de la chambre sociale de la Cour de cassation des 14 novembre 2000 , 22 janvier 2003 et 3 novembre 2004, qui énoncent que l'insuffisance de résultats ne constitue pas en soi une cause réelle et sérieuse de licenciement).

(21) On constate peu d'évolution de la structure des établissements par taille d'effectifs, c'est pourquoi nous étudions la possibilité d'effets de structure pour la seule variable du secteur d'activité. des changements de pratiques des employeurs en matière de rupture pour motif économique.

Notre analyse a d'abord révélé la place importante des démissions et des ruptures conventionnelles dans les fins de CDI à côté des deux motifs de licenciement, puis nous avons émis des hypothèses sur les liens susceptibles d'exister entre les évolutions de chacune de ces ruptures. Afin d'aller plus loin, tentons à présent de saisir s'il existe des relations entre les usages que font les employeurs des modes de rupture. En effet, même si les démissions et, dans une moins mesure, les ruptures conventionnelles, ne dépendent pas des employeurs qui n'en sont pas nécessairement à l'initiative, elles peuvent néanmoins avoir un impact sur la manière dont, stratégiquement, ils gèrent leur main-d'œuvre. Par exemple, on peut imaginer que lorsque les démissions sont nombreuses dans un établissement, cela peut diminuer d'autant le besoin de licencier de l'employeur.

\section{L'usage des modes de rupture révèle-t-il des pratiques particulières de gestion de la main-d'œuvre?}

Étudier l'usage des modes de rupture dans une logique de gestion de la main-d'œuvre, entendue comme un ensemble de modes d'entrées (CDD, $\mathrm{CDI}$ ) et de sorties (licenciements, démissions, départs à la retraite, fins de $\mathrm{CDD}$, etc.) mis à disposition des entreprises (PICHON, 2008), peut permettre de faire apparaître des groupes particuliers d'établissements, selon leur niveau général de rotation de la main-d'œuvre en CDI ou même en CDD.

\section{Une apparente segmentation des établissements selon les pratiques de gestion de la main-d'œuvre des employeurs}

Les établissements ayant recours aux licenciements sont-ils également caractérisés par une présence plus forte de démissions ou même un recours plus important aux CDD ? Pour répondre à cette question, nous avons d'abord créé une typologie d'établissements selon leur recours exclusif ou conjoint aux deux motifs de licenciement et l'avons confrontée ensuite à l'usage des démissions. D'après cette typologie, $64 \%$ des établissements d'au moins dix salariés n'ont recouru en réalité ni au LME, ni au LMP au cours de l'année 2010(22), ce qui est cohérent avec la faible diffusion de leur usage observée précédemment. À l'inverse, la part des établissements ayant recouru conjointement aux deux motifs de licenciement durant l'année

(22) Nous ne considérons ici que la seule année 2010, les proportions d'établissements de chaque catégorie de la typologie s'étant peu modifiées sur la période d'ensemble 1997-2010. Les données en évolution sont disponibles sur demande auprès de l'auteure. 
Graphique 6 : Liens entre les pratiques de licenciement et l'usage de la démission en 2010, selon la taille d'effectifs

Catégories de licenciement et présence des démissions

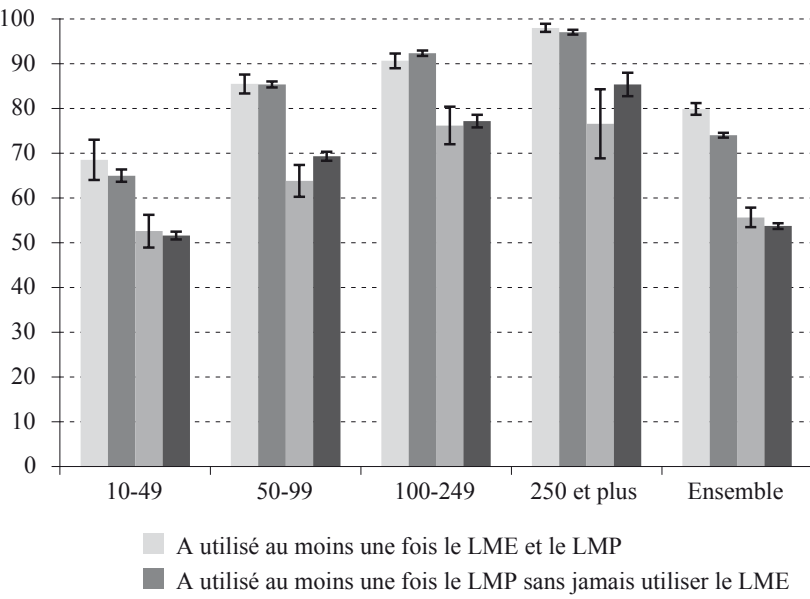

Catégories de licenciement et intensité des démissions (si présence)

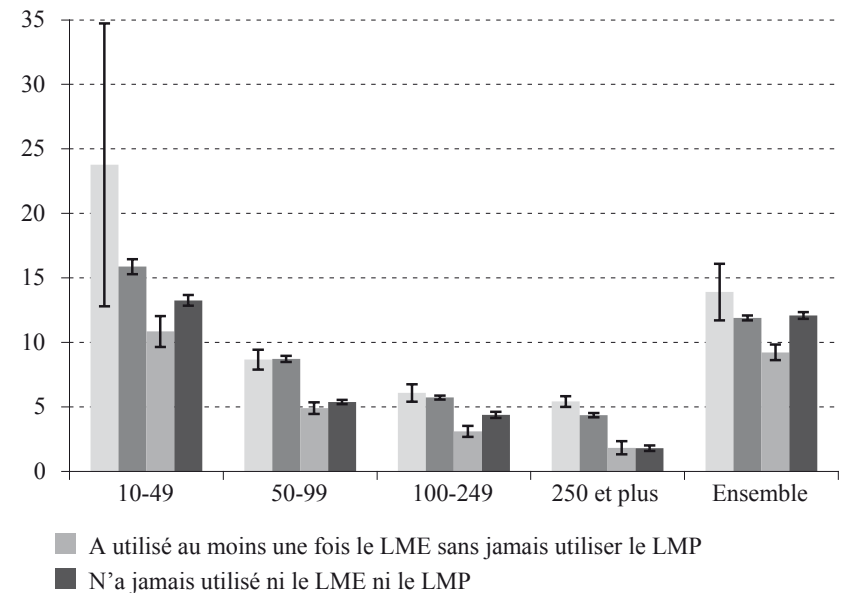

Note : Les intervalles de confiance à $95 \%$ des moyennes sont indiqués sur chaque barre d'histogramme.

Champ : Établissements de dix salariés ou plus du secteur concurrentiel (hors agricole) de la France métropolitaine.

Source : Calculs de l'auteure à partir des EMMO-DMMO, Dares, données pondérées.

2010 est très faible $(3 \%)$ et l'a toujours été sur l'ensemble de la période (6\% en 1997). Lorsqu'ils utilisent au moins un des deux motifs de licenciement, la situation la plus fréquente est celle où les établissements ne recourent qu'au LMP au cours de l'année (30\% en 2010), ce qui est là encore cohérent avec la répartition des deux motifs de licenciement dans les fins de CDI. Enfin, parce que les LME sont caractérisés par une faible diffusion générale dans les établissements, le recours exclusif au LME est lui aussi assez rare (3\% en 2010). Dans la suite de l'analyse, nous nous focalisons sur les deux pratiques de licenciement que sont l'usage conjoint des deux motifs et l'usage exclusif du LMP, car l'utilisation exclusive du LME paraît aujourd'hui très spécifique.

Dans une optique de stratégie de gestion de la main-d'œuvre établie à partir de l'ensemble des modes de sorties auxquels l'entreprise a recours, on pourrait supposer que le faible usage observé des licenciements est compensé par une présence plus importante des démissions dans les établissements, permettant de maintenir un certain niveau de rotation de la main-d'œuvre en CDI, assuré dans ce cas essentiellement par des départs volontaires des salariés. Suivant cette logique, les établissements où il n'y a pas de licenciement devraient avoir un usage plus fort des démissions. En réalité, on observe le contraire dans les graphiques ci-dessus (voir colonnes «Ensemble» sur le graphique 6) : ce sont davantage les établissements utilisateurs de licenciement (des deux motifs ou du LMP seul) que les non-utilisateurs où l'on observe également des démissions (graphique de gauche); en revanche, il ne semble pas y avoir de différences significatives dans l'intensité des démissions entre les trois principales catégories d'établissements selon leur usage du licenciement (graphique de droite).

La complémentarité entre usage du licenciement, notamment pour motif personnel, et présence de démissions pourrait être surtout expliquée par un «effet taille» des établissements, puisque l'on a vu que la taille influait fortement sur l'intensité et le recours aux modes de rupture (voir supra). Le graphique 6 montre toutefois que le lien de complémentarité entre la présence de démissions dans les établissements et le recours aux licenciements se retrouve bien pour toutes les tailles d'effectifs (graphique de gauche). C'est pour l'indicateur d'intensité que le résultat est le plus intéressant (graphique de droite). Excepté pour les établissements de petite taille qui ne se distinguent pas de l'ensemble (23), un lien apparaît de nouveau entre l'usage des licenciements et l'intensité des démissions, ce pour les tailles d'effectifs supérieures à cinquante salariés. Le résultat de l'ensemble est donc expliqué essentiellement par les petits établissements qui sont majoritaires dans l'échantillon, mais qui sont également sous-représentés dans les deux principales catégories d'usage des licenciements (LME et LMP, LMP seul).

Finalement, cette différenciation des établissements selon leur pratique de rupture du CDI se maintient si l'on intègre la rotation des CDD (entrée et sorties). Le graphique 7 montre en effet que les établissements caractérisés par une forte rotation de

(23) Pour les petits établissements, l'indicateur d'intensité des démissions apparaît très imprécis pour ceux qui recourent conjointement au moins une fois au LME et au LMP au cours de l'année 2010. Cela s'explique par le fait que le croisement de ces deux caractéristiques, petite taille et usage conjoint des motifs de licenciement, concerne en réalité un très petit nombre d'établissements. 
la main-d'œuvre en $\mathrm{CDI}^{(24)}$ ont également un taux élevé de rotation en CDD. La hiérarchie dans le niveau de rotation en CDI (fort, moyen, faible) est d'ailleurs en lien avec celle des taux de rotation en CDD.

\section{Graphique 7 : Évolution du taux de rotation en CDD} selon le niveau de rotation en CDI

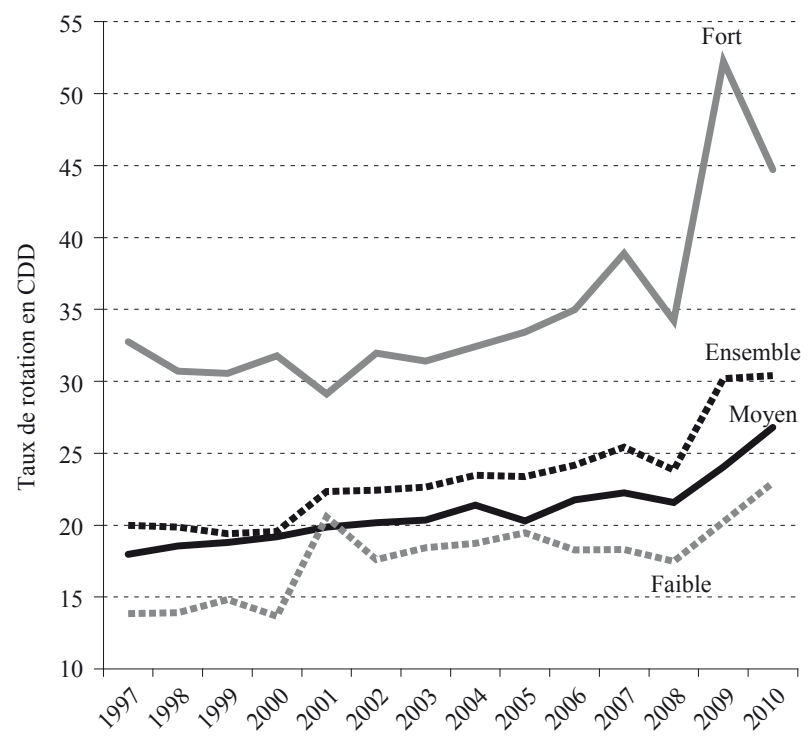

Note : Un taux de rotation en CDI «fort» signifie ici qu'il est supérieur au troisième quartile de l'ensemble de la distribution, «moyen» qu'il est entre le troisième et le premier quartile, et «faible» qu'il est inférieur au premier quartile.

Lecture : En 2010, les établissements caractérisés par un fort taux de rotation en CDI avaient un taux de rotation en CDD de $45 \%$, bien supérieur au taux moyen de l'ensemble des établissements de $30 \%$.

Champ : Établissements de dix salariés ou plus du secteur concurrentiel (hors agricole) de la France métropolitaine.

Source : Calculs de l'auteure à partir des EMMO-DMMO, Dares, données pondérées.

Ces résultats soutiennent l'idée d'une segmentation des établissements selon leur pratique de rotation de la main-d'œuvre, des complémentarités plus que des substitutions existant entre usage des ruptures de CDI et de CDD. Ce lien positif entre le recours aux CDD et l'usage des LMP ou encore des démissions, avait déjà été démontré au début des années 1990 pour les établissements de 50 salariés et plus, les auteurs interprétant ce lien comme la «conséquence d'un affaiblissement des relations d'emploi au sein de l'organisation» (LE Minez, Maurin, 1994, p. 7). L'apparition de la rupture conventionnelle peut-elle alors remettre en cause cette segmentation, en révélant des nouvelles opportunités de rupture pour les établissements qui n'avaient jusque-là qu'une faible rotation de leur main-d'œuvre?

\section{Quels liens entre l'usage de la RC et les autres modes de gestion de la main-d'œuvre?}

Le tableau suivant résume les liens entre l'usage de la RC, tant en intensité qu'en recours, et les pratiques de licenciement, de démission et, plus largement, de rotation en CDI. De manière générale, il ressort des données un lien positif entre l'usage des ruptures de CDI déjà existantes et celui de la RC. Ce sont les catégories d'usage conjoint des deux motifs de licenciement, d'usage conjoint du licenciement et de la démission, et de taux de rotation en CDI (hors RC) fort, qui ont un recours à la RC plus élevé. Au contraire, les établissements qui n'ont jamais recouru au LME et au LMP au cours d'une année donnée, ou même connu des démissions, sont caractérisés par un recours plus faible à la RC.

Cependant, l'étude des liens de ces catégories d'établissements avec l'intensité de la RC nous invite à nuancer ces premiers constats ${ }^{(25)}$. En effet, si l'on retrouve un tel lien positif entre le niveau de rotation en CDI qualifié de «fort» et le taux de RC pour les établissements utilisateurs, des taux de RC relativement élevés sont également observés pour les établissements ayant une rotation en CDI faible, ou pour ceux n'ayant pas connu dans l'année ni démission ni licenciement (tous motifs confondus). Ce résultat étaierait l'argument selon lequel de nouvelles opportunités de rupture du CDI s'offriraient aux établissements jusqu'ici peu utilisateurs. Peut-être la RC ne s'estelle pas seulement substituée à des ruptures déjà existantes, mais a-t-elle aussi permis la conclusion de ruptures qui n'auraient sinon pas eu lieu. Un tel résultat rejoint celui d'une étude antérieure (Signoretto, 2013b).

L'introduction de la rupture conventionnelle dans le paysage des ruptures du CDI n'a visiblement pas substantiellement modifié la segmentation des établissements selon leur niveau de rotation de la main-d'œuvre en CDI, même si des pratiques différentes relatives à l'intensité à laquelle les établissements recourent à la $\mathrm{RC}$ sont observées. Du côté de l'usage des CDD, des tests supplémentaires non reproduits ici, ne montrent pas de différence significative des taux de rotation en CDD selon le recours ou non à la RC en 2009 et en 2010 .
(24) Le taux de rotation est défini comme suit: (taux d'entrée + taux de sortie)/2. Pour les sorties de CDI, sont pris en compte les licenciements, les démissions, les départs à la retraite et les RC à partir de 2009.
(25) La précision statistique de l'indicateur d'intensité de la RC n'étant pas toujours assurée, l'interprétation des résultats du tableau doit être réalisée en tenant compte des intervalles de confiance de la moyenne de ces taux. 
Tableau : Lien entre les ruptures du CDI déjà existantes (licenciements, démissions) et la RC

\begin{tabular}{|c|c|c|c|c|c|c|c|c|c|c|c|}
\hline & \multicolumn{4}{|c|}{2009} & \multicolumn{4}{|c|}{2010} & \multicolumn{2}{|c|}{ Ensemble } \\
\hline & & \multirow{2}{*}{ 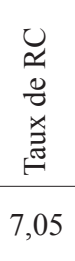 } & \multirow{2}{*}{ 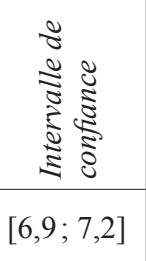 } & \multirow{2}{*}{ 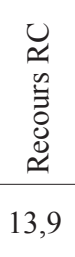 } & \multirow{2}{*}{ 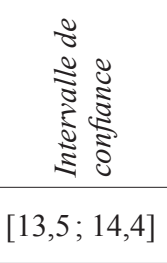 } & \multirow{2}{*}{ 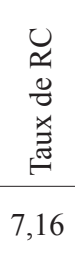 } & \multirow{2}{*}{ 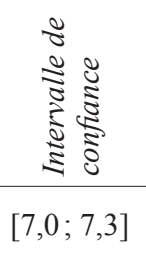 } & \multirow{2}{*}{ 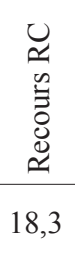 } & \multirow{2}{*}{ 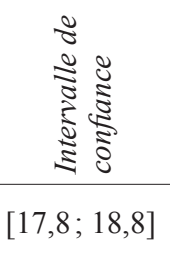 } & \multirow{2}{*}{ 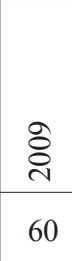 } & \multirow{2}{*}{$\begin{array}{l}\stackrel{\circ}{\circ} \\
64\end{array}$} \\
\hline \multirow{4}{*}{ 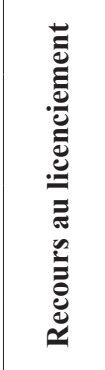 } & $\begin{array}{l}\text { N'a jamais recouru ni au } \\
\text { LME ni au LMP }\end{array}$ & & & & & & & & & & \\
\hline & $\begin{array}{l}\text { A recouru au moins une } \\
\text { fois au LME sans jamais } \\
\text { recourir au LMP }\end{array}$ & 5,79 & {$[5,5 ; 6,1]$} & 15,7 & {$[14,3 ; 17,2]$} & 5,78 & {$[5,4 ; 6,2]$} & 24,1 & {$[22,2 ; 26,0]$} & 5 & 3 \\
\hline & $\begin{array}{l}\text { A recouru au moins une } \\
\text { fois au LMP sans jamais } \\
\text { recourir au LME }\end{array}$ & 4,55 & {$[4,5 ; 4,7]$} & 20,2 & {$[19,8 ; 20,7]$} & 4,38 & {$[4,3 ; 4,5]$} & 27,3 & {$[26,8 ; 27,8]$} & 31 & 30 \\
\hline & $\begin{array}{l}\text { A recouru au moins une } \\
\text { fois au LME et au LMP }\end{array}$ & 5,81 & {$[5,3 ; 6,3]$} & 34,4 & {$[33,1 ; 35,7]$} & 6,86 & {$[5,7 ; 8,1]$} & 41,2 & {$[39,6 ; 42,8]$} & 4 & 3 \\
\hline \multirow{4}{*}{ 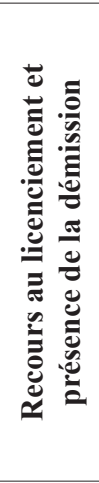 } & $\begin{array}{c}\text { N'a jamais connu ni } \\
\text { de démission ni de } \\
\text { licenciement }\end{array}$ & 7,67 & {$[7,4 ; 7,9]$} & 13,2 & {$[12,5 ; 13,9]$} & 7,94 & {$[7,6 ; 8,3]$} & 15,3 & {$[14,5 ; 16,0]$} & 30 & 29 \\
\hline & $\begin{array}{l}\text { A connu au moins une fois } \\
\text { une démission sans jamais } \\
\text { recourir au licenciement }\end{array}$ & 6,48 & {$[6,3 ; 6,7]$} & 14,6 & {$[14,0 ; 15,2]$} & 6,67 & {$[6,5 ; 6,8]$} & 20,9 & {$[20,3 ; 21,6]$} & 30 & 34 \\
\hline & $\begin{array}{l}\text { A recouru au moins une } \\
\text { fois au licenciement sans } \\
\text { jamais avoir connu une } \\
\text { démission }\end{array}$ & 5,89 & {$[5,7 ; 6,1]$} & 15,1 & {$[14,6 ; 16,0]$} & 6,02 & {$[5,8 ; 6,3]$} & 19,1 & {$[17,9 ; 20,1]$} & 13 & 10 \\
\hline & $\begin{array}{c}\text { A connu au moins une } \\
\text { fois une démission et un } \\
\text { licenciement }\end{array}$ & 4,58 & {$[4,5 ; 4,7]$} & 24,0 & {$[23,5 ; 24,4]$} & 4,51 & {$[4,3 ; 4,7]$} & 31,6 & {$[31,1 ; 32,2]$} & 27 & 26 \\
\hline \multirow{4}{*}{ 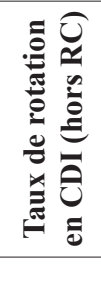 } & Faible & 5,86 & {$[5,7 ; 6,0]$} & 13,9 & {$[13,3 ; 14,5]$} & 5,45 & {$[5,3 ; 5,6]$} & 16,3 & {$[15,7 ; 16,9]$} & 25 & 25 \\
\hline & Moyen & 5,22 & {$[5,1 ; 5,3]$} & 17,2 & {$[16,8 ; 17,6]$} & 4,94 & {$[4,9 ; 5,0]$} & 22,1 & {$[21,6 ; 22,6]$} & 50 & 50 \\
\hline & Fort & 7,30 & {$[7,0 ; 7,6]$} & 19,0 & {$[18,3 ; 19,7]$} & 8,20 & {$[7,8 ; 8,6]$} & 27,1 & {$[26,3 ; 28,0]$} & 25 & 25 \\
\hline & Ensemble & 6 & {$[5,9 ; 6,1]$} & 17 & {$[16,5 ; 17,1]$} & 6 & {$[5,9 ; 6,2]$} & 22 & {$[21,6 ; 22,2]$} & 100 & 100 \\
\hline
\end{tabular}

Note : Seules les années 2009 et 2010 sont représentées dans le tableau. La RC n'a commencé à être effective qu'à partir du milieu de l'année 2008 ; par conséquent, nous ne disposons pas de données permettant une bonne représentativité des pratiques des établissements cette année-là. Champ : Établissements de dix salariés ou plus du secteur concurrentiel (hors agricole) de la France métropolitaine.

Source : Calculs de l'auteure à partir des EMMO-DMMO, Dares, données pondérées.

Cette approche par un usage de l'ensemble des modes de rupture du CDI mis en lien avec le recours aux CDD montre qu'en réalité c'est pour un groupe particulier d'établissements, caractérisés par un fort taux de rotation (en CDI et en CDD), que les pratiques de gestion de la main-d'œuvre semblent particulièrement sensibles aux règles juridiques encadrant les modes de rupture du CDI. Cette segmentation des établissements pourrait être ultérieurement précisée en fonction du secteur d'activité(26), ainsi que de la situation économique, car ces facteurs pourraient expliquer un besoin de rotation de la main-d'œuvre plus fort dans certains établissements.

(26) BOURIEAu et al. (2014) montrent notamment que le secteur du tertiaire est caractérisé par un taux de rotation largement supérieur aux deux autres secteurs.
La focalisation sur l'évolution opposée des deux motifs de licenciement comme fait principal diffusé dans la littérature économique sur l'usage des règles de protection de l'emploi à durée indéterminée ne semble pas justifiée. En effet, elle apparaît seulement sur une courte période et n'est plus visible quand on élargit la fenêtre d'observation. Mais surtout, elle masque un autre phénomène qui constitue la régularité empirique principale des trois dernières décennies: le recours au licenciement pour motif économique, comme forme juridique de rupture, est en baisse continue sur longue période et ne concerne plus qu'une minorité d'établissements. Si des difficultés d'identification statistique dans les sources de données peuvent expliquer une partie de cette baisse, c'est surtout des changements de pratiques de gestion de la main-d'œuvre en matière 
de suppressions d'emplois qui sont à l'œuvre. Or, cette diversité des ruptures pour motif économique résulte d'un usage par les employeurs de dispositifs légaux ${ }^{(27)}$ ou de possibilités reconnues par la jurisprudence ${ }^{(28)}$, et non d'un contournement de règles juridiques. C'est dans cette même logique que l'usage de la rupture conventionnelle par les employeurs et les salariés doit être analysé. Apparaissant parfois plus opportune aux employeurs et/ou aux salariés, elle peut se substituer aux autres modes de rupture du CDI - sans que l'on puisse estimer toutefois dans quelle proportion - puisqu'elle a élargi les possibilités offertes par la loi en la matière. Plus généralement, la prise en compte de l'ensemble des modes de rupture du CDI et de leurs interactions a permis d'identifier des pratiques segmentées d'employeurs : ce sont les mêmes établissements caractérisés par une présence forte de démissions qui licencient et concluent plus souvent des ruptures conventionnelles. L'usage des règles juridiques de rupture du CDI impacte d'abord ces établissements qui ont vraisemblablement besoin d'une rotation de la main-d'œuvre plus forte que d'autres, ce qui pourrait s'expliquer par un environnement économique plus fluctuant ou une volatilité sectorielle de la demande plus forte. Ce résultat incite à creuser ce que révèle plus précisément cette segmentation des établissements en fonction de la manière dont ils mettent en œuvre leur politique de gestion des ressources humaines.

\section{Bibliographie}

ARNold C. (2009), «Les mouvements de main-d'œuvre en 2008 : un infléchissement après un début d'année en hausse», Premières informations, premières synthèses, $\mathrm{n}^{\circ}$ 44.2, Paris, Dares.

Beaujolin-Bellet R., Schmidt G. (2012), Les restructurations d'entreprises, Paris, La Découverte.

Bourieau P., Paraire X., Sanzeri O. (2014), «Les mouvements de main-d'œuvre en 2013. Forte augmentation des entrées en CDD dans le tertiaire», Dares analyses, $\mathrm{n}^{\circ} 094$.

Cahuc P., Kramarz F. (2004), De la précarité à la mobilité: vers une Sécurité sociale professionnelle, Rapport au ministre de l'Économie, des Finances et de l'Industrie et au ministre de l'Emploi, du Travail et de la Cohésion sociale, Paris, La Documentation française.

Colin J.-F., Welcomme D. (1981), «L'employabilité des demandeurs d'emploi. Une description à partir des statistiques de 1'ANPE», Travail et emploi, $\mathrm{n}^{\circ}$ 9, pp. 31-46.

García-Martínez P., Malo M. À. (2007), «The strategic use of dismissal legislation: an empirical analysis using Spanish data», European journal of law and economics, vol. 23, n 2 , pp. 151-167.

INSTITUT DE RECHERCHES ÉCONOMIQUES ET SOCIALES (IRES) (2009), La France du travail. Données, analyses, débats, Ivry-sur-Seine, Éd. de l'Atelier, Éditions ouvrières.

Lagarenne C., Le Roux M. (2006), «Les licenciements en 2003 : trois fois plus nombreux pour motif personnel que pour raisons économiques », Premières informations, premières synthèses, $\mathrm{n}^{\circ} 11.1$, Paris, Dares.

(27) Tels les dispositifs d'accompagnement des restructurations, les résiliations amiables pour motif économique, les préretraites.

(28) Comme la reconnaissance de la licéité des ruptures conventionnelles pour une «cause économique».
Le Minez S., MaURin É. (1994), «Recours aux contrats à durée déterminée : une relation d'emploi plus fragile, mais moins de licenciements économiques 》, Premières synthèses, ${ }^{\circ} 45$, Paris, Dares.

LUTINIER B. (2007), «Séries de données régionales sur les mouvements de main-d'œuvre entre 1996 et 2005 », Document d'études, $\mathrm{n}^{\circ} 124$, Paris, Dares.

Lyon-Caen A., Affichard J. (dir.) (2008), L'évaluation $d u$ droit du travail : problèmes et méthodes, Rapport final, vol. I, Rapport de synthèse, Paris, Institut international pour les études comparatives (IIPEC), Appel à projets Dares «L'analyse économique du droit du travail».

NoËL F. (1995), Licenciements et démissions: la régulation des effectifs sous contrat à durée indéterminée, mémoire de DEA de l'université de Paris-IX Dauphine.

Organisation DE COOPÉRATION ET DE DÉVELOPPEMENT ÉCONOMiQues (OCDE) (2004), Perspectives de l'emploi de l'OCDE 2004, Paris, OCDE.

Palpacuer F., Seignour A., Vercher C. (2005), Le licenciement pour motif personnel: un dispositif juridique au carrefour des mutations contemporaines de la relation d'emploi, Rapport réalisé pour le compte de la Dares.

Pichon A. (2008), «Le contrat à durée indéterminée (CDI) dans la tourmente de l'entreprise flexible», $L a$ Revue des sciences de gestion, $\mathrm{n}^{\circ} 230$, pp. 53-60.

Pignoni M.-T., Zouary P. (2003), «Les nouveaux usages $\mathrm{du}$ licenciement pour motif personnel», Premières informations, premières synthèses, $\mathrm{n}^{\circ} 28.2$, Paris, Dares.

SAUZE D. (2003), «La progression des CDD et leur rôle dans les variations de l'emploi en France de 1985 à 2000 », in Euzeby C., Carluer F., Chapon S., Euzeby A., Mansanti D., Offredi C. (éd.), Mondialisation et régulation sociale, t. 2, XXIII's journées de l'Association d'économie sociale, Paris, L'Harmattan, pp. 825-840. 
Serverin É., Valentin J., Kirat T., Sauze D., Dalmasso R. (2008), «Évaluer le droit du licenciement. Comparaison des droits et des procédures, mesure des actions », Revue de l'OFCE, n 107, pp. 29-64.

Signoretto C. (2013a), Licenciements et rupture conventionnelle: analyse et évaluation empirique des comportements des employeurs, Thèse de doctorat en sciences économiques, Université Paris-1 Panthéon-Sorbonne.

Signoretto C. (2013b), «Rupture conventionnelle, destructions d'emplois et licenciements : une analyse empirique sur données d'entreprises (2006-2009)», Documents de travail du Centre d'économie de la Sorbonne, $\mathrm{n}^{\circ} 2013.69$.

Signoretto C. (2015, à paraître), «Restructurations, gestion de l'emploi et droit du travail: analyse institutionnelle et statistique », Revue de l'Ires, $\mathrm{n}^{\circ} 84$.

Tomasini M., Le Roux M. (2002), «Diminution des licenciements économiques en 2000», Premières synthèses, $\mathrm{n}^{\circ} 13.1$, Paris, Dares.

\section{Annexe}

Tableau : Recours et intensité de l'usage des licenciements et des démissions entre 1997 et 2010 par secteur d'activité

\begin{tabular}{|c|c|c|c|c|c|c|c|c|c|c|c|c|c|}
\hline \multirow[t]{2}{*}{ En \% } & \multicolumn{3}{|c|}{ Industrie } & \multicolumn{3}{|c|}{ Construction } & \multicolumn{3}{|c|}{ Tertiaire } & \multicolumn{3}{|c|}{ Ensemble } & \multirow{2}{*}{$\begin{array}{c}\text { Évolution } \\
\text { sans } \\
\text { changement } \\
\text { de structure }\end{array}$} \\
\hline & 1997 & 2010 & Évolution & 1997 & 2010 & Évolution & 1997 & 2010 & Évolution & 1997 & 2010 & Évolution & \\
\hline \multicolumn{14}{|c|}{ Licenciement pour motif économique } \\
\hline Intensité (si recours) & 10,01 & 10,98 & $10 \% *$ & 10,94 & 11,66 & $7 \% *$ & 10,05 & 9,42 & $-6 \% *$ & 10,15 & 10,07 & $-1 \% *$ & $-1 \%$ \\
\hline Recours & 18,1 & 11,0 & $-39 \%$ & 17,1 & 5,1 & $-70 \%$ & 12,2 & 5,2 & $-57 \%$ & 14,2 & 6,2 & $-56 \%$ & $-53 \%$ \\
\hline \multicolumn{14}{|c|}{ Licenciement pour motif personnel } \\
\hline Intensité (si recours) & 5,01 & 4,66 & $-7 \%$ & 6,99 & 6,67 & $-5 \% *$ & 7,14 & 6,84 & $-4 \%$ & 6,56 & 6,46 & $-2 \% *$ & $-4 \%$ \\
\hline Recours & 31,9 & 34,0 & $6 \%$ & 27,2 & 35,1 & $29 \%$ & 28,9 & 32,8 & $13 \%$ & 29,5 & 33,2 & $13 \%$ & $13 \%$ \\
\hline \multicolumn{14}{|l|}{ Démission } \\
\hline Intensité (si présence) & 8,59 & 8,46 & $-1 \% *$ & 10,31 & 9,17 & $-11 \%$ & 14,68 & 13,10 & $-11 \%$ & 12,85 & 12,02 & $-6 \%$ & $-10 \%$ \\
\hline Présence & 51,6 & 54,6 & $6 \%$ & 48,9 & 58,1 & $19 \%$ & 55,3 & 62,4 & $13 \%$ & 53,7 & 60,7 & $13 \%$ & $12 \%$ \\
\hline Part du secteur d'activité & 24 & 16 & $-33 \%$ & 10 & 11 & $5 \%$ & 65 & 73 & $11 \%$ & & & & \\
\hline
\end{tabular}

Note : * signifie que l'évolution ne semble pas significative au regard des intervalles de confiance à $90 \%$ des moyennes entre les deux années correspondantes.

Champ : Établissements de dix salariés ou plus du secteur concurrentiel (hors agricole) de la France métropolitaine.

Source : Calculs de l'auteure à partir des EMMO-DMMO, Dares, données pondérées. 